\title{
Narrativa transmedia y representación mediática: el caso de \#Luimelia
}

\author{
Transmedia storytelling and media representations: the case of \\ \#Luimelia
}

\section{Transmídia narrativa e representação midiática: o caso de \#Luimelia}

Mercedes Herrero De la Fuente, Grupo de investigación Nebrija_INNOMEDIA. Universidad Antonio de Nebrija, Madrid, España (mherrero@nebrija.es)

Anto Garzía, Universidad Antonio de Nebrija, Madrid, España (agarciado@nebrija.es)

María-José Establés, Grupo de investigación Nebrija_INNOMEDIA. Universidad Antonio de Nebrija, Madrid, España (mestables@nebrija.es)

RESUMEN | Esta investigación analiza el impacto en las redes sociales del spin-off \#Luimelia, emitido en febrero-marzo de 2020 como una extensión de narrativa transmedia de una de las tramas de la ficción española Amar es para siempre, la más vista a diario en España. Los fans bautizaron con esta etiqueta la relación afectivoamorosa entre dos mujeres, visibilizando de forma positiva las relaciones lésbicas en una serie de ficción. Eléxito de su historia da lugar a un nuevo producto transmedia, que fomenta las sinergias entre plataformas y convierte a las redes sociales en un elemento narrativo más. A partir del análisis cuantitativo de su repercusión en Twitter y de tres entrevistas en profundidad semiestructuradas a los dos guionistas de la serie y a una profesional responsable de la medición de audiencias, se revela cómo la movilización de los fans fue decisiva para el surgimiento de \#Luimelia y cómo su estrategia de redes contribuye al desarrollo de la trama y a construir los personajes protagonistas.

PALABRAS CLAVE: narrativa transmedia; redes sociales; Twitter; series de ficción; comunidades de fans; representación mediática; \#Luimelia. 
ABSTRACT / This research analyzes the impact on social networks of the spin-off \#Luimelia, broadcasted in February-March 2020 as a transmedia narrative extension of one of the plots of the Spanish fiction series Amar es para siempre, the most watched daily on television in Spain. Fans baptized with this label the affective-amorous relationship between two women, making lesbian relationships visible in a positive way in a fictional series. The success of their story gives rise to a new transmedia product, which promotes synergies between platforms and turns social networks into another narrative element. Based on the quantitative analysis of its impact on Twitter and three semi-structured in-depth interviews to the two scriptwriters of the series and a professional responsible for the measurement of audiences, as well as the quantitative analysis of its impact on Twitter, we show how the mobilization of fans was decisive for the emergence of \#Luimelia and how its networking strategy contributes to the development of the plot and the construction of the main characters.

KEYWORDS: transmedia storytelling; social networks; fiction series; Twitter; fandom; media representation; \#Luimelia.

RESUMO|Esta investigação analisa o impacto nas redes sociais do spin-off \#Luimelia, transmitida durante fevereiro-março de 2020 como uma extensão narrativa transmédia de uma das tramas da série de ficção espanhola Amar es para siempre, a mais vista diariamente na televisão na Espanha. Os fãs batizaram com este título a relação afetivo-amorosa entre duas mulheres, tornando as relações lésbicas visíveis de uma forma positiva numa série de ficção. O sucesso da sua história dá origem a um novo produto transmídia, que fomenta sinergias entre plataformas e transforma as redes sociais num outro elemento narrativo. Com base na análise quantitativa do seu impacto no Twitter e em três entrevistas em profundidade semiestruturadas com os dois roteiristas da série e uma profissional responsável pela medição da audiência, é revelado como a mobilização dos fãs foi decisiva para a emergência da \#Luimelia e como a sua estratégia de rede contribui para o desenvolvimento do enredo e para a construção das personagens principais.

PALAVRAS-CHAVE: narrativa transmídia; redes sociais; Twitter; série de ficção; comunidades de fãs; representação na mídia; \#Luimelia. 


\section{INTRODUCCIÓN}

En el actual ecosistema mediático las series de ficción son uno de los proyectos que más consumen los jóvenes, tanto a través de plataformas digitales de video bajo demanda como en redes sociales (Rubio-Jordán, 2021; García-Orta et al., 2019; Martínez Forteza et al., 2019; Raya et al., 2018). Sus creadores piensan cada vez más en la lógica de ampliar las narrativas sin perder de vista los fines comerciales, proponiendo historias que se amplían, se matizan o simplemente se cuentan de manera diferente (Varona Aramburu \& Lara Toledo, 2015).

Este artículo se centra en el universo transmedia generado en torno de la primera temporada de la serie \#Luimelia (ATRESplayer PREMIUM), emitida en febrero-marzo de 2020. Su éxito conduce a una segunda temporada entre agosto y septiembre del mismo año, seguida de dos más durante 2021. Procede de una de las tramas de la serie original Amar es para siempre ${ }^{1}$, sobre la relación entre dos personajes mujeres, Luisita Gómez y Amelia Ledesma, que se extiende y cobra vida en la plataforma de pago de Atresmedia ${ }^{2}$ y a través de las redes sociales.

\#Luimelia es un ejemplo, junto con Veneno, de la apuesta de esta compañía por añadir contenidos sobre personajes con identidades sexuales no heteronormativas. Ambas han sido nominadas en la 32a edición de los premios GLAAD (Seif, 2021), la principal organización internacional que aboga por la representación de las personas lesbianas, homosexuales, transexuales, bisexuales, intersexuales y queers (LGTBI) en los medios de comunicación.

También ha funcionado como precedente para otras series españolas, como Acacias 38, que replica un romance entre dos lesbianas (Maite y Camino), proyectada en redes como \#Maitino. Ejemplos en series latinoamericanas son \#Jemma y \#Aristemo en Argentina y \#Juliantina en México (Acosta, 2020).

\section{MARCO TEÓRICO: NARRATIVAS TRANSMEDIA Y REPRESENTACIÓN EN LA FICCIÓN DE LAS RELACIONES HOMOSEXUALES ENTRE MUJERES}

Distintos autores caracterizan las narrativas transmedia (Establés, 2020; Guerrero-Pico \& Scolari, 2016; Jenkins, 2003, 2006; Jenkins et al., 2013; Scolari, 2014;

1. Es una de las series estrella de Antena 3 desde 2013. Antes, durante siete temporadas se emitió en Radio Televisión Española, con el título Amar en tiempos revueltos.

2. Atresmedia: nombre de marca de Atresmedia Corporación de Medios de Comunicación, S.A, grupo español que opera en varios sectores, especialmente el audiovisual. Cuenta con dos principales cadenas de televisión generalista en abierto, Antena 3 y La Sexta. Tiene habilitada la plataforma de video bajo demanda ATRESplayer PREMIUM. 
Scolari et al., 2012; Scolari \& Establés, 2017; Scolari \& Ibrus, 2014) y distinguen cuatro elementos fundamentales en estas:

- La historia debe expandirse a través de diferentes medios o plataformas (series de televisión, libros, videojuegos, etc.). Es fundamental que cada medio cuente de la mejor forma posible la historia mientras aporta contenido novedoso, ampliándose así el universo narrativo.

- Las expansiones pueden ser gestionadas de diferentes formas. La expansión desde arriba es por parte de los creadores (top-down). La desde abajo (bottom-up) surge de los usuarios.

- Las narrativas transmedia se basan en la capacidad del universo narrativo de ser abordado desde cualquiera de los textos que lo componen. Por lo tanto, entre ellos tienen cierta autonomía.

- La teoría de los dos tipos de desarrollo de una narrativa transmedia distingue entre estrategia y táctica (Scolari et al., 2012). Un diseño estratégico implica que una serie es ideada desde los guiones iniciales como un producto transmediático.

Los estudios sobre la representación de las relaciones amorosas entre mujeres en la industria audiovisual muestran que estas tienden a reproducir el cliché de la hot lesbian (Jackson \& Gilbertson, 2009). Ese arquetipo de las relaciones lésbicas ha estado más vinculado con "la construcción de un objeto de deseo destinado a complacer una mirada masculina heterosexual que a ofrecer un modelo de referencia sobre el cual las mujeres lesbianas pudieran sentirse identificadas" (Guerrero-Pico et al., 2017, p. 32).

Otro de los clichés asociados es el del síndrome de la lesbiana muerta (Acosta, 2020; Guerrero-Pico et al., 2017; Guerrero-Pico et al., 2018; Tosenberger, 2008b; Waggoner, 2018), en el cual la consumación de una relación afectivo-amorosa entre mujeres es castigada en muchas ficciones con la muerte violenta de alguna de las mujeres de la pareja (Ventura et al., 2019).

Otros estudios de caso sobre ficciones televisivas (Establés-Heras \& Rivera Pinto, 2015; Guerrero-Pico, 2017; Jenkins, 2015; Rodrigues Lima \& Cavalcanti, 2020; Tosenberger, 2008b) han analizado cómo el fandom incide en las representaciones tradicionales, sobre todo el que está muy implicado en la creación de contenidos y tiene una actitud activista que pueda reclamar la renovación de las series.

Las comunidades de fans de algunos productos audiovisuales se suelen caracterizar por defender y reivindicar una visibilización positiva de diferentes tendencias sexuales, así como por normalizar personajes que no son 
heteronormativos mediante diferentes trabajos transformativos, como fanfics, fanarts o espacios seguros digitales para compartir conversaciones, impresiones y aprendizajes (Brennan, 2014, 2018; Condis, 2015; Masanet \& Buckingham, 2015; Day \& Christian, 2017; De Kosnik, 2016; Pande \& Moitra, 2017; Tosenberger, 2008b; De los Ríos Izquierdo, 2021a; García Muñoz, 2021). Esto ocurre particularmente en el caso de las comunidades de fans en las que las mujeres son mayoría (De los Ríos Izquierdo, 2021b; Villa-Montoya et al., 2019; Brennan, 2014; Bacon-Smith, 1992).

De hecho, en los últimos años estos discursos reivindicativos provenientes de los fandoms han trascendido sus espacios habituales. Se puede apreciar que las series de ficción con personajes no heteronormativos están adquiriendo más protagonismo, tanto en las cadenas tradicionales de televisión como en las plataformas de video bajo demanda (Day \& Christian, 2017; González de Garay et al., 2019; McNicholas Smith \& Tyler, 2017; Ng, 2017; Raya et al., 2018; Symes, 2017).

\section{\#Luimelia como narrativa transmedia estratégica}

La elección de \#Luimelia ${ }^{3}$ como objeto de estudio se centra en dos pilares fundamentales: se trata de una narrativa transmedia estratégica, con fines comerciales para la emisora, y supone un producto audiovisual que aporta una visión positiva de las relaciones afectivo-amorosas entre mujeres.

La serie cumple con las cuatro características de una narrativa transmedia, aprovechada como producto de la cultura fandom por la propia industria cultural como un nuevo producto. En efecto, utiliza dos medios diferentes: la plataforma de pago ATRESplayer PREMIUM para emitir la serie, y Twitter e Instagram.

Por otra parte, los creadores promueven la interacción continua entre la serie y los contenidos publicados en las redes sociales, buscando la retroalimentación de los fans, amplificando determinadas tramas. Se incluyen además argumentos no aparecidos en los capítulos emitidos en la plataforma digital.

La historia de Luisita y Amelia se puede seguir de forma independiente tanto en redes sociales como en ATRESplayer PREMIUM, y se puede decir que esta serie ha tenido un diseño estratégico, pues fue ideada desde los guiones iniciales como un producto transmediático.

Es una creación impulsada por una audiencia joven que simpatiza con esta trama de la serie primigenia. A través del shippeo creado por los fans surge la etiqueta \#Luimelia, convertida en trending topic y que derivará posteriormente a la

3. \#Luimelia, en cursiva, alude específicamente a la serie; en letra redonda denomina el conjunto de la estrategia transmedia. 
expansión transmedia, con una serie propia. El shippeo se refiere a "la implicación romántica de dos personajes de una obra de ficción y/o celebridades. Se puede tratar de relaciones que estén establecidas de forma canónica dentro del universo narrativo; relaciones ambiguas que se están desarrollando o relaciones que es improbable que sucedan. El típico shipping es la creación de una palabra nueva compuesta por la unión de los nombres de los personajes" (Establés, 2020, p. 154). Por tanto, el universo narrativo de Amar es para siempre y de \#Luimelia se rige por las lógicas de las narrativas transmedia.

\section{METODOLOGÍA}

Como se afirmó, el objetivo principal de esta investigación es analizar la estrategia en redes sociales que interactúa con la emisión de la serie \#Luimelia en ATRESplayer PREMIUM.

Para observar las características de \#Luimelia, analizar su estrategia de narrativa transmedia en redes sociales y dimensionar su repercusión, buscamos responder a las siguientes interrogantes:

- ¿Cómo Luisita y Amelia pasan de ser personajes de una trama de la serie convencional Amar es para siempre a protagonizar un nuevo producto transmedia con gran eco en redes sociales?

- ¿Han influido las acciones de los fans en el surgimiento de \#Luimelia?

- ¿Existen determinadas sinergias entre la estrategia de redes sociales y la serie \#Luimelia?

- ¿Contribuyen los perfiles virtuales en Twitter de Luisita y Amelia a narrar su historia como pareja y a construir esos personajes sirviendo de apoyo a la serie?

- ¿\#Luimelia tiene algún impacto en superar los prejuicios en torno a las lesbianas?

Se optó por una metodología mixta. El abordaje cuantitativo se centra en una muestra de Twitter, por ser la red social con posibilidades de medición más avanzadas, incluyendo referencias muy puntuales a Instagram. La medición se divide en dos periodos. El primero se centra en la séptima temporada de Amar es para siempre (septiembre 2018 - agosto 2019). El segundo analiza la primera temporada de \#Luimelia (desde la fase de promoción, el 12 de diciembre de 2019 y durante su emisión en ATRESplayer PREMIUM, del 14 de febrero al 15 de marzo). Nuestra medición incluyó una semana después del último capítulo. 


\begin{tabular}{cc} 
Indicador & Naturaleza cuantitativa/cualitativa \\
\hline Número de seguidores & Cuantitativa \\
\hline Número total de tuits & Cuantitativa \\
\hline Media diaria de autores & Cuantitativa \\
\hline Número de impresiones totales & Cuantitativa \\
\hline Número total de comentarios & Cuantitativa \\
\hline 20 hashtags más recurrentes & Cuantitativa/cualitativa \\
\hline Trending topics & Cuantitativa/cualitativa \\
\hline Palabras clave & Cualitativa \\
\hline
\end{tabular}

Tabla 1. Principales indicadores del análisis Fuente: Elaboración propia.

Se seleccionan indicadores usados habitualmente para valorar el éxito de una determinada estrategia en Twitter (tabla 1).

En ambos casos accedimos a los datos del equipo de medios sociales de Atresmedia. Sus mediciones se realizaron sobre una muestra de 398.334 tuits, correspondientes a la séptima temporada de Amar es para siempre (frente a los 35.676 de la temporada anterior) (https://www.instarsocial.com/) y sobre un segundo conjunto de 263.901 tuits generados durante la primera temporada de \#Luimelia (https://www.instarsocial.com/). Algunos indicadores resultan de la propia red, pero la mayoría se obtienen con la herramienta Instar Social ${ }^{4}$. Igualmente se han podido consultar informes de seguimiento de las series Amar es para siempre, \#Luimelia y documentos del equipo de medios sociales de Atresmedia.

La dimensión cualitativa de esta investigación está dada por tres componentes:

- La información obtenida de Twitter de los hashtags recurrentes, trending topics y palabras clave, referidos a las creaciones analizadas.

- Una breve guía de estilo de Atresmedia para publicar en los perfiles de Twitter de Luisita y Amelia, que definen las pautas que las caracterizan tanto en el lenguaje, en la expresividad y como usuarias de esta red.

- Tres entrevistas semiestructuradas realizadas con consentimiento informado en julio y agosto de 2020 a sendos profesionales conocedores de la estrategia transmedia de \#Luimelia: Nuria Cano, responsable de Producción y Servicio al

4. Recurso de pago de Kantar (empresa multinacional de estudios de audiencia social líder en España), ofrece el número de impresiones reales. No se trata, por tanto, de un cálculo potencial. 
Cliente Social Media de Kantar (entrevista vía correo electrónico el 27 de julio y completada con nuevos datos y comentarios por el mismo canal los días 28 y 29), y Diana Rojo y Borja González, creadores y guionistas de la serie \#Luimelia (entrevistados de forma presencial y simultánea el 10 de agosto). Nuestro propósito fundamental era analizar si la estrategia de redes ha contribuido a expandir el universo de \#Luimelia en los términos definidos por los creadores y guionistas de la serie, con especial atención a la construcción de los personajes.

\section{ANÁLISIS DE LOS RESULTADOS}

Los resultados de esta investigación describen cómo ha sido la repercusión de \#Luimelia en redes sociales desde distintos ángulos, como las sinergias entre Amar es para siempre y \#Luimelia o el protagonismo de Twitter en la estrategia transmedia de \#Luimelia, con especial atención a su valor narrativo.

\section{Amar es para siempre y \#Luimelia: datos de impacto y sinergias}

En cuanto al impacto de \#Luimelia en la evolución en Twitter de Amar es para siempre, se observa que la trama sobre la relación entre Luisita y Amelia en la serie transforma la repercusión en redes sociales de esta ficción convencional. Los fans crean en Twitter el shippeo \#Luimelia y los datos de la audiencia social se disparan. El primer tuit que acuña el mencionado shippeo demanda a la productora un final feliz entre ambas.

Basta con acudir a los resultados de la temporada anterior para corroborar el impacto del fenómeno \#Luimelia. En 2017-2018 Amar es para siempre acumuló un total de 35.676 tuits, 60 autores de media al día y 8.181.672 impresiones totales. Estas cifras mejoraron considerablemente en 2018-2019, llegando a 398.334 tuits (+1,017\%), 237 autores de media al día (+295\%) y 83.955.420 impresiones totales (+926\%) (Kantar, 2020). Asimismo, la trama relacionada con Luisita y Amelia alcanzó una gran proyección, aumentando un $1000 \%$ en los comentarios en Twitter respecto de la temporada anterior, según afirma Nuria Cano (comunicación personal, 27 de julio de 2020).

Otras valoraciones adicionales (https://www.instarsocial.com/) nos permiten ratificar este éxito: del total de impresiones en la temporada 2018-2019, más de 40 millones hacen referencia a esta trama; "Luimelia" es la palabra clave más usada en Twitter al hablar de la serie (51,6\%), incluso por encima de "amaresparasiempre" (41,3\%); 15 de los 20 hashtags más utilizados están relacionados con este shippeo (entre ellos podemos destacar: \#carolrovira; \#paulausero5; \#luisita; \#amelia; \#lgtb; \#luimeliaorgullo o \#luimeliaparasiempre) y los fans generan trending topics aludiendo a la pareja casi a diario, según el argumento del correspondiente capítulo (Kantar, 2020).

5. Carol Rovira es la actriz que interpreta el papel de Amelia. Paula Usero es su pareja, Luisita. 


\title{
Porfavor @antena3com, @A3AmarSiempre y @atresmediacom, como gran fan que soy de amar es para siempre, os pido y os ruego que @PaulaUsero (Luisita) y Amelia acaben juntas PORFAVOR \#Luimelia
}

\author{
23 Nov $2018 \cdot 21: 57$
}

\section{Imagen 1. Primer tuit con el hashtag referido al shippeo \#Luimelia}

Fuente: Twitter6.

Como afirma Cano, si hablamos de social TV, tal vez el resultado más llamativo de la séptima temporada de Amar es para siempre son los casi 400.000 comentarios generados.

\footnotetext{
"El caso de Amar es para siempre es sorprendente porque rompe con todas las tendencias del social TV. Es una serie diaria, en la que la historia transcurre lentamente y los momentos importantes o resolutivos tardan varios capítulos en acontecer. Además, se emite en un horario donde su competencia directa es uno de los programas más comentados diariamente (Sálvame) y su audiencia es un público senior (70\% de los espectadores son mayores de 55 años). El volumen de comentarios es sostenido durante todo el día, aunque buena parte de ellos se concentra durantela emisión, ya que los usuarios continúan debatiendo y generando contenido sobre la serie a lo largo del día. En mi opinión, es un rotundo éxito de social TV, tanto si lo analizas en datos absolutos como en relativos" (comunicación personal, 27 de julio de 2020).
}

La explosión en redes sociales es acompañada de un incremento de la audiencia televisiva: la cuota de pantalla pasa de $11,9 \%$ a $12,1 \%(+0,2 \%)$. Destaca especialmente el crecimiento en las franjas de edad más jóvenes, que normalmente no son público de estas ficciones diarias: en el tramo de 13 a 15 años sube de 5,1\% a 6,2\% (+1,1\%); de 16 a 24 años, de $7,9 \%$ a $8,6 \%(+0,7 \%)$ y de 25 a 29 años pasa de $9,8 \%$ a 11,5\% $(+1,7 \%)$;(Kantar, 2020).

En lo narrativo, resulta oportuno destacar la interacción entre la serie primigenia y la estrategia transmedia de \#Luimelia. En la nueva serie sus protagonistas son fans de Amar eternamente, que también recoge entre sus tramas el amor entre dos mujeres: Lucía y Aurelia. Además de recoger este argumento (en el tercer capítulo

6. Por protocolo ético se ha anonimizado esta imagen, para proteger la identidad del usuario en Twitter. Esta práctica metodológica se recomienda en el ya citado Guerrero-Pico, et al., 2018. 
de la primera temporada), el interés por este romance salta a los perfiles en Twitter de Luisita y Amelia. La primera expresa con especial vehemencia sus emociones mientras sigue la serie ficticia y adopta en ocasiones una postura crítica (reprocha a los guionistas que censuren las escenas de sexo, por ejemplo). Pero sobre todo, acuña el shippeo \#Lurelia (procedente de Lucía y Aurelia) y pide un spin-off para esta historia de amor. Así, \#Lurelia pasa a figurar entre los 20 hashtags más recurrentes (https://www.instarsocial.com/) en la posición 17, situándose \#jastalurelia en la 15. Ambos términos son registrados igualmente entre las palabras clave aparecidas en Twitter a lo largo de la primera temporada de \#Luimelia. Se recrea por lo tanto una situación real y se alude de esta forma a la génesis de la ficción audiovisual \#Luimelia que, como sabemos, se origina a partir de Amar es para siempre.

Siguiendo con la conexión argumental entre estas dos creaciones, algunos personajes de Amar es para siempre aparecen en \#Luimelia, ya sea en sus capítulos o interactuando en las redes. Se utilizan los falsos compartidos para darles presencia en Twitter e Instagram y así se introduce en la historia de Luisita y Amelia a sus amigos y familiares. Los vínculos entre Amar es para siempre y \#Luimelia explotados por los guionistas se reproducen en la estrategia de redes sociales, amplificando un juego que homenajea a la creación primigenia y funciona como un reclamo para los fans de ambas series.

\section{\#Luimelia como creación transmedia. Protagonismo de las redes sociales}

La serie \#Luimelia comienza a emitirse el 14 de febrero de 2020 en la plataforma de pago de videos bajo demanda ATRESplayer PREMIUM. Es parte de un entorno transmedia que se completa con una detallada estrategia en redes sociales (Twitter e Instagram), iniciada el 12 de diciembre de 2019. Nuestra investigación pone el foco en este segundo elemento, pero contempla todo el conjunto, destacando las sinergias entre las plataformas, las innovaciones narrativas y el impacto registrado en un fandom entusiasmado con \#Luimelia.

Para Nuria Cano, "el spin off de \#Luimelia es vanguardia de la televisión social (...). Antena 3 ha sabido monetizar el interés en redes sociales de una trama, apostando por crear un contenido que gustaba a su público en Twitter, y derivarlo a su plataforma streaming" (comunicación personal, 27 de julio de 2020).

\section{1. \#Luimelia: la serie en ATREStresplayer PREMIUM}

La primera temporada de la comedia romántica \#Luimelia en televisión está formada por seis capítulos de aproximadamente diez minutos de duración, con una emisión semanal. La periodicidad define en gran medida la estrategia transmedia, para generar sinergias entre los capítulos emitidos y las redes sociales, de domingo en domingo (salvo la primera entrega, un viernes). 
A diferencia de Amar es para siempre, que ha ido avanzando en el tiempo hasta los años setenta ${ }^{7}, \#$ Luimelia está ambientada en 2020 , desvela una trama actual y sus protagonistas presentan inquietudes propias de nuestra época. Uno de los retos para los guionistas fue realizar ese trasvase de forma creíble y sin perder la idiosincrasia de ambos personajes. En palabras de Rojo, creadora y guionista, en esta ocasión "contaron con la ventaja de no estar cohibidos por la época" (comunicación personal, 3 de agosto de 2020). Liberados de esa limitación, tuvieron que pensar muy bien determinados aspectos para saltar a nuestros días, con la precaución de no perder a Luisita y Amelia en ese proceso en el que intervienen caracterización, forma de hablar, decorados o iluminación.

Los creadores de \#Luimelia afirman haberse sentido libres para contar su historia sin presiones por el funcionamiento de las redes. Rojo declara: "Escribimos el guion sin estar supeditados a las redes, pero las tenemos in mente (...)”. Borja González añade: "Sabemos que hay tramas que en Twitter o Instagram se potencian" (comunicación personal, 3 de agosto de 2020).

\section{La contribución de Twitter a \#Luimelia}

El alcance en esta red es notable (https://www.instarsocial.com/). \#Luimelia acumula un total de 10 trending topics en Twitter entre febrero y marzo de 2020, 263.901 tuits, más de 70.628 .739 impresiones reales y una media diaria de 507 autores (12 diciembre 2019 - 22 marzo 2020). Sus contenidos promocionales en las redes sociales de @ATRESplayer funcionan de media diez veces mejor que el resto. Por último; \#Luimelia ostenta la tercera posición en la clasificación de programas más comentados (febrero y marzo 2020).

Todo lo anterior ratifica el efecto viral de \#Luimelia en Twitter, con un impacto que se evidencia incluso antes de su emisión. A principios de febrero de 2020 acumulaba 14 millones de impresiones en sus hitos de promoción (enero y febrero 2020) y \#LuimeliaEstreno era el trending topic en segunda posición.

En cuanto al valor narrativo de Twitter, opera como un factor de expansión de la trama, de modo que el devenir de la relación sentimental entre Luisita y Amelia se mantiene vivo en sus publicaciones más allá de los argumentos contados semanalmente en ATRESplayer PREMIUM. Para el desarrollo en redes resulta decisivo no ofrecer los capítulos simultáneamente. Ello permite trasladar la narración más allá del marco de la emisión dominical a otros espacios donde están los potenciales seguidores (Twitter e Instagram). Para ello, se utilizan los perfiles

7. Comienza en los primeros años del franquismo. Tras diez temporadas, llega a los momentos previos a la transición democrática. Su antecedente, Amar en tiempos revueltos, se desarrolla sobre todo durante la guerra civil española (1936-391939) y la posguerra. 
de las dos protagonistas, quienes tienen una voz propia en las redes, aportando un elemento de cercanía y activando la conversación con los fans. Lo anterior constituye un factor distintivo de la estrategia en redes sociales de \#Luimelia.

Estas avanzan a la misma velocidad que los episodios, de modo que entre un capítulo y otro existe un acompañamiento. Sin embargo, el rodaje se grabó en un breve espacio de tiempo (entre noviembre y diciembre de 2019), periodo aprovechado por el equipo de medios sociales para generar materiales que pudieran utilizar más tarde (explotando los decorados, el atrezo, la caracterización de los personajes, etc.) en la fase de emisión (febrero y marzo de 2020).

La estrategia transmedia de \#Luimelia explota las posibilidades de las redes para generar una retroalimentación con las líneas argumentales de cada capítulo. Se parte del análisis de los guiones para decidir qué elementos de la trama se pueden potenciar y diversificar. La narración evoluciona de forma integrada en ambos universos, de manera que la serie no incluye todo lo que acontece y las redes se utilizan para presentar determinados contenidos exclusivos, recrearse en algunos detalles del argumento, matizar a los personajes e intensificar la comunicación con el fandom, donde se percibe una presencia clara del colectivo LGTBI.

Los creadores y guionistas de \#Luimelia declaran ser conscientes de las posibilidades de las redes para extender tramas. González ilustra: "Es como cuando cocinas con alguien. Si le pone un toque de algo que te gusta, lo usas la próxima vez que hagas ese plato. Si en redes surge algo y nos gusta, los guionistas lo incorporamos. Es una sinergia muy importante" (comunicación personal, 3 de agosto de 2020).

Incidiendo en la función narrativa de Twitter, detectamos dos características fundamentales: el carácter multimodal de esta red con la plataforma donde se emite la serie \#Luimelia y su contribución para construir los personajes protagonistas. Las abordamos a continuación.

En cuanto a lo primero, destacamos aquí algunos de los elementos más representativos utilizados para este diálogo entre la serie y las redes en Twitter.

- La aparición de Amar eternamente en el guion de \#Luimelia y sus redes. Su argumento se desarrolla en paralelo al de la serie, estableciéndose una sincronización entre ambas: "Jugamos a que los que estaban viendo Amar es para siempre contaran con la interacción en redes de Luisita, que estaba siguiendo la serie ficticia", explica González (comunicación personal, 3 de agosto de 2020). Las críticas en Twitter por censurar las escenas de sexo son recogidas en uno de los capítulos de \#Luimelia, que finaliza con una escena de ese tipo. El director dice "corten" y en ese momento el personaje de Luisita 
se rebela, exige que la acción continúe y los espectadores pueden ver cómo las dos mujeres hacen el amor. Así, el cierre se rectifica y se hace un guiño a un elemento narrativo amplificado en redes. Rojo afirma: "Luisita está muy enganchada a Amar eternamente y este es un tema que ha continuado hasta hoy, más allá del capítulo centrado en esta trama" (comunicación personal, 3 de agosto de 2020).

- En conexión con lo anterior, un segundo instrumento de interacción es la controversia en torno al famoso síndrome de la lesbiana muerta (Deshler, 2017; Guerrero-Pico et al., 2017, 2018), que refiere al final dramático de numerosas series sobre relaciones entre lesbianas. Esta es lanzada en las redes por Luisita, quien expresa su rechazo por el desenlace traumático en Los hombres de Paco de la historia de amor entre una mujer policía y su novia, quien muere en una emboscada en sus propios brazos (Gómez, 2020). Esto muestra empatía con el colectivo LGTBI, implicando un giro en el guion de la serie.

- Se recurre a un discurso multimodal entre la serie y las redes para anunciar la segunda temporada de \#Luimelia, dando visibilidad en Twitter a un personaje, Toñi, vecina de las protagonistas. Su existencia se desprende en determinados momentos de la serie, a pesar de que nunca aparece. Toñi cobra relevancia en esta red mediante un video, referido a las conversaciones entre Luisita y Amelia que escucha desde su casa. Este contenido en clave humorística recuerda el término manzanilla, parte de un juego de palabras que los fans identificaban con el primer encuentro amoroso de la pareja. Asimismo, en esta retroalimentación entre la plataforma y las redes, Luisita expresa en alguna ocasión en Twitter su disgusto por los golpes que la vecina da en su pared. Los seguidores asocian a Toñi con un rostro que solo se visibiliza en el entorno virtual.

- En esta incorporación a Twitter de elementos narrativos ausentes en la primera entrega de la serie destacamos los tuits aparecidos en el perfil de Amelia, pero escritos por Luisita, cuando se apropia del teléfono móvil de su pareja. Los fans detectan esta intrusión, porque reconocen su estilo impetuoso y esto provoca numerosos comentarios. Los guionistas rentabilizan esta anécdota discurrida en las redes para incorporarla en la segunda temporada, en el capítulo 2. En otro ejemplo Luisita y Amelia se besan en los baños de un conocido local madrileño. Son sorprendidas por la hermana de Luisita, quien supuestamente las graba y publica el video en Twitter. Estas acciones en redes proporcionan verosimilitud a la historia, activan la participación de los fans y destacan determinadas características de los personajes. 
La segunda característica fundamental de la estrategia en redes es la preocupación por captar la esencia de las protagonistas. Los guionistas presentan a dos mujeres reales, con las que -más allá de su orientación sexual- prácticamente cualquiera puede empatizar, lo que es recogido en sus perfiles. Rojo observa: "Es fácil identificarse con ellas, porque tienen sus defectos y eso es interesante para empatizar. Poseen una química muy cotidiana, por ello cualquier persona en una relación de pareja, sea lesbiana o heterosexual, se puede sentir identificada" (comunicación personal, 3 de agosto de 2020).

Así, Luisita y Amelia se comportan en redes como lo haría cualquiera, al compartir contenidos de amigos, interactuar con personajes famosos a los que siguen, mostrar su indignación o celebrar su alegría, e incluso participar en eventos reales.

Un ejemplo son las publicaciones del 8 de marzo, día de la Mujer Trabajadora, con ambas asistiendo a la gran manifestación celebrada en Madrid, o las del día del orgullo LGTBI, cuando cada una de ellas posa con la bandera multicolor.

Según Rojo, "Luisita es una chica muy impulsiva; tiene claro lo que quiere, pero mete bastante la pata, se equivoca y luego tiene que pedir perdón" (comunicación personal, 3 de agosto de 2020). Refiriéndose a Amelia, González destaca como sus rasgos más relevantes ser "soñadora, sensible, emotiva, fiel (...). Soñadora y sensible, sobre todo" (comunicación personal, 3 de agosto de 2020). Los perfiles virtuales reflejan a la perfección la personalidad de cada una. Una variedad de recursos, desde la redacción de los textos a la utilización de determinados elementos visuales, contribuyen a definir a las protagonistas y acercarlas a su público.

Los personajes en redes tienen su propia guía de estilo definida por el equipo de medios sociales de Atresmedia, que describen los siguientes aspectos: su forma de escribir, el tipo de contenido que publican, los elementos con los que destacan sus publicaciones, y la pericia con la que manejan las redes. Esta guía es un espejo de los prototipos representados por Luisita y Amelia. La primera es torpe en su manejo; escribe jasta porque no sabe lo que es un hashtag, redacta sin puntuar, reflejando una forma de expresarse impulsiva, publica textos poco elaborados en los que dice lo que piensa sin remilgos y usa pocos recursos visuales, salvo el emoticón recurrente de un croissant (revela así un aspecto de sus gustos) y algunas fotos, a veces movidas o mal encuadradas.

Amelia es actriz y utiliza sus perfiles para proyectarse profesionalmente. Pretende dar una imagen sofisticada. Cuida la redacción, selecciona contenidos que le parece deben interesar a una artista, maneja filtros y su interacción no es espontánea. Comparte música como reggaetón, aunque en la serie descubrimos que lo que de verdad le gusta es la copla. 
no entiendo el jasta \#felizlunes si es el día que menos nos gusta a todos pero bueno que seáis felices todos jasta \#lurelia

1:53 p. m. $\cdot 24$ feb. $2020 \cdot$ Twitter for iPhone

\section{Imagen 2. Tuit de Luisita que ejemplifica su uso de Twitter}

Fuente: Gómez (2020).

Amelia Ledesma

@AmeliaLedesmaOf

"No puedes rendirte nunca. Los ganadores nunca se rinden y los que se rinden nunca ganan".

Casting a casting, siempre adelante. En unos dias tengo otro \#AmeliaLedesma \#Castings \#NewChallenges

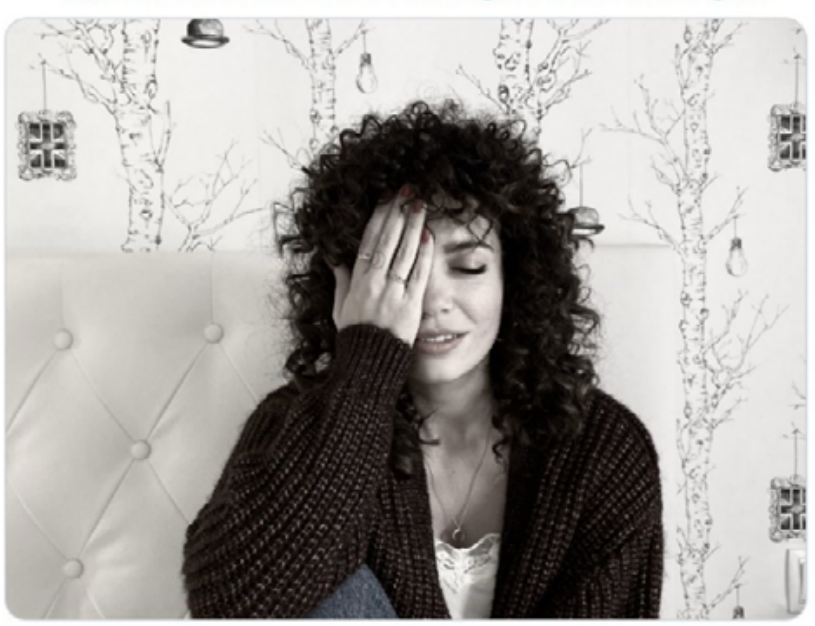

11:24 p. m. $\cdot 27$ feb. 2020 - Twitter for iPhone

Imagen 3. Tuit de Amelia que ejemplifica su uso de Twitter Fuente: Ledesma (2020).

Los datos de estos perfiles muestran su éxito tras la primera temporada, pues acumulan más de 29 mil seguidores (Twitter e Instagram; febrero y marzo 2020) y más de ocho millones de impresiones en las publicaciones generadas solo en estas cuentas, como muestra la tabla 2 (https://www.instarsocial.com; febrero y marzo 2020). 


\begin{tabular}{cccc} 
Seguidores & Twitter & Instagram & Total \\
\hline @LuisitaGomezS & 5187 & 9644 & 14.831 \\
\hline @AmeliaLedesmaOf & 4977 & 9474 & 14.451 \\
\hline Total & 10.164 & 19.118 & 29.282 \\
\hline
\end{tabular}

Tabla 2. Seguidores de la serie en Twitter e Instagram

Fuente: Elaboración propia con base en datos de Instar Social (https://www.instarsocial.com), marzo 2020.

Para concluir con el rol de Twitter en el desarrollo de estos personajes, es fundamental señalar cómo se aborda su condición de lesbianas. Se trata de una aproximación libre de prejuicios y alejada de los estereotipos negativos que normalmente se asocian con ellas y con sus relaciones. La intención de los guionistas de presentar sin traumas el amor entre dos chicas jóvenes se proyecta fielmente en las redes: "No queríamos una serie donde se señalara que por ser una pareja de lesbianas van a tener necesariamente determinados problemas. Creo que la demanda de representación desde el colectivo LGTBI lo que pide sobre todo es normalizar sus relaciones", subraya Rojo (comunicación personal, 3 de agosto de 2020). González suscribe: "Nuestra intención era reflejar la relación entre Luisita y Amelia de la mejor manera. Y para nosotros, eso era contar esta historia igual que contaríamos cualquier otra" (comunicación personal, 3 de agosto de 2020).

Esta empatía es valorada por el colectivo LGTBI, como se refleja en los hashtags más recurrentes, donde \#luimeliagaypride figura en el puesto 19 (https://www. instarsocial.com/). Se recoge de esta forma una tendencia registrada en la séptima temporada de Amar es para siempre, en la que \#lgtb y \#luimeliaorgullo aparecían entre las etiquetas más frecuentes (https://www.instarsocial.com/).

La comunicación fluida con la audiencia LGTBI se constata en numerosas publicaciones, en las que sus miembros celebran la relación de esta pareja o expresan vivencias personales relacionadas con su orientación sexual.

Pero \#Luimelia presenta una proyección más amplia, conectando con una audiencia que simpatiza con la causa LGTBI pero que, sobre todo, se siente atraída por el devenir de una historia romántica. Prueba de ello es que fuera de lo mencionado más arriba las mediciones de Instar Social no detectan en Twitter otros términos relacionados con este colectivo en los trending topics, las etiquetas más recurrentes o las palabras clave de la primera temporada de \#Luimelia. 
Las publicaciones de Luisita y Amelia en Twitter poseen, por lo tanto, un impacto positivo en la representación mediática de las lesbianas, que aleja a estas mujeres y sus relaciones de prototipos retrógrados y favorece la empatía de una audiencia más amplia hacia el colectivo LGTBI. Existen multitud de ejemplos entre los tuits publicados por las protagonistas y las respuestas de sus fans que lo respaldan. Señalamos aquí algunos que muestran la felicidad de la pareja y aportan una imagen de cada una totalmente ajena al prototipo masculinizado que tradicionalmente ha acompañado a las lesbianas ${ }^{8}$.

\section{CONCLUSIONES}

En esta investigación hemos indagado sobre el singular proceso que da lugar a \#Luimelia. Su carácter novedoso radica en el contexto en que se produce el fenómeno fan en torno a la pareja formada por Luisita y Amelia, parte de Amar es para siempre, serie veterana y convencional de emisión diaria, seguida por un espectador tipo mayor de 55 años. Sin embargo, y rompiendo con las tendencias de la social TV, la relación entre ambas mujeres multiplica el impacto en redes sociales de la ficción en su séptima temporada y genera un fandom que a partir del shippeo \#Luimelia culmina en una nueva serie. Tras detectar el éxito de la trama y potenciarla, principalmente en Twitter, Atresmedia canaliza su gran popularidad en una nueva creación transmedia a principios de 2020.

Lo relevante del nuevo producto es la eficacia con la que se utilizan los distintos recursos narrativos, en especial en la estrategia de redes sociales. Esta se basa en los perfiles virtuales de ambas protagonistas, que desde Twitter e Instagram generan nuevos argumentos, amplían determinadas tramas, interactúan con otros personajes, se relacionan con los fans con especial sensibilidad por el colectivo LGTBI (Contreras Pulido et al., 2020) y hacen continuos guiños sobre determinados leitmotiv, entre ellos la serie ficticia Amar eternamente (en homenaje a Amar es para siempre).

8. Ledesma, A. [@AmeliaLedesmaOf].(2020, marzo 7). Estando a tu lado nada puede ir malamente [Tweet]. Twitter. https://twitter.com/AmeliaLedesmaOf/status/1236390217227141125 Gómez, L. [@LuisitaGomezS]. (2020, agosto 8). qué guapa mi novia [Tweet]. Twitter. https://twitter.com/LuisitaGomezS/status/1292131038798458883 Gómez, L. [@LuisitaGomezS]. (2020, marzo 2). muy orgullosa de ti, te quiero [Tweet]. Twitter. https://twitter.com/LuisitaGomezS/status/1234591197882179584 Gómez, L. [@LuisitaGomezS]. (2020, septiembre 6). amore te quiero [Tweet]. Twitter. https://twitter.com/LuisitaGomezS/status/1302598970578001922 Ledesma, A. [@AmeliaLedesmaOf]. (2020, marzo 8). \#AmeliaLedesma \#love \#party [Tweet]. Twitter. 
La serie \#Luimelia y el entorno de las redes sociales se retroalimentan dentro de una narrativa ágil, que enriquece la historia y contribuye también a perfilar los propios personajes. Ambas protagonistas reflejan su personalidad en el uso que hacen de Twitter e Instagram y se desenvuelven como cualquier chica de su edad, compartiendo momentos especiales, expresando su alegría o demostrando indignación. Así, se profundiza en la construcción de estos arquetipos en la expansión transmedia, cuya característica más destacada es probablemente el hecho de tratarse de dos mujeres, con las que una gran parte de la audiencia se puede ver identificada de forma positiva, evitando clichés como el del síndrome de la lesbiana muerta (Cameron, 2018; Deshler, 2017; Guerrero-Pico et al., 2017; Guerrero-Pico et al., 2018; Tosenberger, 2008a; Waggoner, 2018).

Lo anterior es probablemente el principal aporte de \#Luimelia al colectivo LGTBI, específicamente a una representación mediática de las lesbianas que rompe con los prototipos negativos que rodean sus relaciones. Es decir, Luisita y Amelia sitúan su relación dentro de la normalidad.

Esta capacidad para generar empatía convierte a \#Luimelia en un fenómeno de gran magnitud, respaldado por una amplia comunidad fandom muy activa, con iniciativa y habilidad para generar trending topics. Su alto impacto en redes sociales, desde la etapa en que surge de Amar es para siempre, se revalida al estrenarse como nueva serie en ATRESplayer PREMIUM. Su repercusión sigue creciendo en el momento de publicarse este artículo, tras su segunda temporada, en agostoseptiembre de 2020, y de emitir dos entregas más en 2021.

Futuras líneas de trabajo podrían indagar en dos aspectos fundamentales. El primero se refiere a la estrategia transmedia de la segunda temporada, centrada en desarrollar discursos multimodales en redes sociales. El segundo se focaliza en los fandoms, especialmente en las acciones desarrolladas a raíz de la representación positiva de las relaciones amorosas entre personajes LGBTI en series de ficción, como se discutió en el marco teórico.

\section{REFERENCIAS}

Acosta, M. (2020). La acción conectiva por la visibilización de la diversidad sexual en el fenómeno comunicativo \#Luimelia (Connective action for the visibility of sexual diversity in the communicative phenomenon \#Luimelia). IC - Revista Científica de Información y Comunicación, (17), 533-558. https://doi.org/10.32870/cys.v2020.7312

Bacon-Smith, C. (1992). Enterprising women: television fandom and the creation of popular myth. University of Pennsylvania Press

Brennan, J. (2014). 'Fandom is full of pearl clutching old ladies': Nonnies in the online slash closet. International Journal of Cultural Studies, 17(4), 363-380.

https://doi.org/10.1177/1367877913496200 
Brennan, J. (2018). Queerbaiting: The 'playful' possibilities of homoeroticism. International Journal of Cultural Studies, 21(2), 189-206. https://doi.org/10.1177/1367877916631050

Cameron, K. (2018). Toxic regulation: From TV's code of practices to '\#Bury Your Gays.' Participations: Journal of Audience \& Reception Studies, 15(1), 1-14. http://www.participations.org/Volume 15/Issue 1/18.pdf

Condis, M. (2015). No homosexuals in Star Wars? BioWare, "gamer" identity and the politics of privilege in a convergence culture. Convergence: The International Journal of Research into New Media Technologies, 21(2), 198-212. https://doi.org/10.1177/1354856514527205

Contreras Pulido, P., Guzmán Franco, M. D., \& Vizcaíno Verdú, A. (2020). La Bella y la Bestia y el movimiento LGTB en Disney: empoderamiento y activismo en YouTube (Beauty and the Beast and the LGBT movement in Disney: Empowering and Activism on YouTube). Área Abierta, 20(1), 75-93. https://doi.org/10.5209/arab.63129

Day, F. \& Christian, A. J. (2017). Locating Black Queer TV: Fans, Producers and Networked Publics on YouTube. Transformative Works and Cultures, 24(Queer Female Fandom special issue). https://doi.org/10.3983/twc.2017.867

De Kosnik, A. (2016). Rogue Archives. Digital Cultural Memory and Media Fandom. The MIT Press. https://doi.org/10.7551/mitpress/10248.001.0001

De los Ríos Izquierdo, C. (2021a). Fandom, fanwork y aprendizaje no formal en la red (Fandom, fanwork and non-formal learning on the net) (Doctoral dissertation, Universidad Complutense de Madrid). https://eprints.ucm.es/id/eprint/64367/

De los Ríos Izquierdo, C. (2021b). Las mujeres como protagonistas del fanfiction hispanohablante (Women as protagonists of Spanish-speaking fanfiction). FEMERIS: Revista Multidisciplinar De Estudios De Género, 6(3), 33-52. https://doi.org/10.20318/femeris.2021.6402

Deshler, K. M. (2017). Not Another Dead Lesbian: The Bury Your Gays Trope, Queer Grief, and The 100. Whitman College.

Establés, M.-J. (2020). Entre profesionales y aficionados: relaciones y tensiones entre fanse industrias culturales, procesos y estrategias de trabajo y creación (Among professionals and amateurs: relations and tensions between fans and cultural industries, processes and work strategies and creation) (Doctoral dissertation, Universitat Pompeu Fabra). https://84.88.27.106/handle/10803/669211

Establés-Heras, M. J. \& Rivera Pinto, D. (2015). “Ministéricos” en Twitter y WhatsApp, o cómo el poder de los fans puede mover montañas ("Ministerical " on Twitter and WhatsApp, or how the power of fans can move mountains). In C. Cascajosa (Ed.), Dentro del Ministerio del Tiempo (Inside the Time Ministry) (pp. 211-218). Léeme Libros.

García Muñoz, E. (2021). Representación lésbica en las series de TV del siglo XXI. Libro ilustrado de besos entre mujeres (Lesbian representation in 21st century TV series. Illustrated book of kisses between women) (Undergraduate thesis, Universitat Politècnica de València). http://hdl.handle.net/10251/165495

García-Orta, M. J., García-Prieto, V., \& Suárez-Romero, M. (2019). Nuevos hábitos de consumo audiovisual en menores: aproximación a su análisis mediante encuestas (New audiovisual consumption habits among minors: approximantion through the analysis of survey data). Doxa Comunicación. Revista Interdisciplinar de Estudios de Comunicación y Ciencias Sociales, (28), 241-260. https://doi.org/10.31921/doxacom.n28a13 
Gómez, L. [@LuisitaGomezS]. (2020, Febrero 24). No entiendo el jasta \#felizlunes si es el día que menos nos gusta a todos pero bueno que seáis felices todos jasta \#lurelia [Tweet]. Twitter. https://twitter.com/luisitagomezs/status/1231925114079522817

Gómez, L. [@LuisitaGomezS]. (2020, Febrero 23). No me lo puedo creer NO ME LO PUEDO CREER. Otra lesbiana muerta en una serie y encima el día de su boda no puede ser [Tweet]. Twitter. https://twitter.com/AmeliaLedesmaOf/status/1231364786958544896

González de Garay, B., Marcos Ramos, M., \& Sánchez González, S. (2019). Maternidades lésbicas en la ficción televisiva española (Lesbian maternity in spanish tv fiction). Investigaciones Feministas, 10(2), 295-314. https://doi.org/10.5209/infe.66495

Guerrero-Pico, M. (2017). \#Fringe, Audiences and Fan Labor: Twitter Activism to Save a TV Show from Cancellation. International Journal of Communication, 11, 2071-2092. ttps://ijoc.org/index.php/ijoc/article/view/4020

Guerrero-Pico, M., Establés, M. J., \& Ventura, R. (2017). Dead Lesbian Syndrome: LGBTQ fandom's self-regulation mechanisms in fan-producer controversies around The 100. Anàlisi. Quaderns de Comunicaciói Cultura, (57), 29-46. https://doi.org/https://doi.org/10.5565/rev/analisi.3110

Guerrero-Pico, M., Establés, M. J., \& Ventura, R. (2018). Killing off Lexa: ‘Dead lesbian syndrome' and intra-fandom management of toxic fan practices in an online queer community. Participations. Journal of Audience \& Reception Studies, 15(1), 311-333. http://www.participations.org/Volume 15/Issue 1/17.pdf

Guerrero-Pico, M. \& Scolari, C. A. (2016). Narrativas transmedia y contenidos generados por los usuarios: el caso de los crossovers (Transmedia storytelling and user-generated contents: A case study on crossovers). Cuadernos.Info, (38), 183-200. https://doi.org/10.7764/cdi.38.760

Jackson, S. \& Gilbertson, T. (2009). ‘Hot Lesbians’’ Young People’s Talk About Representations of Lesbianism. Sexualities, 12(2), 199-224. https://doi.org/10.1177/1363460708100919

Jenkins, H. (2003, January 15). Transmedia Storytelling. Moving characters from books to films to video games can make them stronger and more compelling. MIT Technology Review. https://www.technologyreview.com/s/401760/transmedia-storytelling/

Jenkins, H. (2006). Convergence culture: where old and new media collide. University Press.

Jenkins, H. (2015). “Cultural Acupuncture”: Fan Activism and the Harry Potter Alliance. In L. Geraghty (Ed.), Popular Media Cultures (pp. 206-229). Palgrave Macmillan. https://doi.org/10.1057/9781137350374_11

Jenkins, H., Ford, S., \& Green, J. (2013). Spreadable media: creating value and meaning in a networked culture. New York University Press.

Kantar. (2020). Anuario Social TV 2019 (Social TV Yearbook 2019). https://www.kantarmedia.com/es/blog-y-recursos/data-lab/anuario-social-tv-2019

Ledesma, A. [@AmeliaLedesmaOf]. (2020, febrero 27). “No puedes rendirte nunca. Los ganadores nunca se rinden y los que se rinden nunca ganan". Casting a casting, siempre adelante. En unos días tengo otro [Tweet]. Twitter. https://twitter.com/ amelialedesmaof/status/1233156046404902915 
Martínez Forteza, A., De Casas Moreno, P., \& Franco Guzmán, M. D. (2019). La alfabetización mediática frente al auge de las nuevas plataformas audiovisuales: consumo y hábitos de la audiencia (Media literacy in the face of the rise of new audiovisual platforms: consumption and audience habits). In P. de Casas Moreno, G. Paramio Pérez, \& V. B. Gómez Pablos (Eds.), Realidades educativas en la esfera digital: sistemas, modelos $y$ paradigmas de aprendizaje (Educational realities in the digital sphere: systems, learning models and paradigms) (pp. 85-104). Ediciones Egregius.

Masanet, M. J. \& Buckingham, D. (2015). Advice on life? Online fan forums as a space for peer-to-peer sex and relationships education. Sex Education, 15(5), 486-499. https://doi.org/10.1080/14681811.2014.934444

McNicholas Smith, K. \& Tyler, I. (2017). Lesbian brides: post-queer popular culture. Feminist Media Studies, 17(3), 315-331. https://doi.org/10.1080/14680777.2017.1282883

$\mathrm{Ng}$, E. (2017). Between text, paratext and context: Queerbaiting and the contemporary media landscape. Transformative Works and Cultures, 24 (Queer female fandom special issue). https://doi.org/10.3983/twc.2017.0917

Pande, R. \& Moitra, S. (2017). "Yes, the Evil Queen is Latina!": Racial dynamics of online femslash fandoms. Transformative Works and Cultures, 24 (Queer female fandom special issue). https://doi.org/10.3983/twc.2017.0908

Raya, I., Sánchez-Labella, I., \& Durán, V. (2018). The construction of the teenager profile on Netflix Tv Shows 13 Reasons Why and Atypical. Comunicación y Medios, (37), 131-143. https://comunicacionymedios.uchile.cl/index.php/RCM/article/view/48631

Rodrigues Lima, C. A. \& Cavalcanti, G. K. (2020). "Homophobia is so last century": activism of telenovela fans in defense of fictional lesbians couples. Comunicación y Sociedad, 1-28. https://doi.org/10.32870/cys.v2020.7312

Rubio Jordán, A. V. (2021). Las series de ficción televisivas en sus webs: análisis de las estrategias transmedia y de la participación activa de la audiencia (Television fiction series on their websites: analysis of transmedia strategies and active audience participation). adComunica. Revista Científica de Estrategias, Tendencias e Innovación en Comunicación, (22), 281-298. https://doi.org/10.6035/2174-0992.2021.22.10

Scolari, C. A. (2014). Narrativas transmedia: nuevas formas de comunicar en la era digital (Transmedia storytelling: new ways of communicating in the digital era). Anuario AC/E de Cultura Digital, 1, 71-81.

Scolari, C. A. \& Establés, M. J. (2017). El ministerio transmedia: Expansiones narrativas y culturas participativas (The Transmedia Ministry: Narrative Expansions and Participatory Cultures). Palabra Clave, 20(4), 1008-1041. https://doi.org/10.5294/pacla.2017.20.4.7

Scolari, C. A. \& Ibrus, I. (2014). Transmedia critical: empirical investigations into multiplatform and collaborative storytelling. International Journal of Communication, 8(1), 2191-2200. https://ijoc.org/index.php/ijoc/article/view/3102

Scolari, C. A., Jiménez, M., \& Guerrero, M. (2012). Narrativas transmediáticas en España: Cuatro ficciones en busca de un destino cross-media (Transmedia storytelling in Spain: four fictions in search of a cross-media destiny). Comunicacion y Sociedad, 25(1), 137-163. https://revistas.unav.edu/index.php/communication-and-society/article/view/36179 
Seif, R. (2021). The Media Representation of Fictional Gay and Lesbian Characters on Television: A Qualitative Analysis of U.S. TV-series regarding Heteronormativity (Doctoral dissertation, Jönköping University). https://www.diva-portal.org/smash/record.jsf?pid=diva2\%3A1111368\&dswid=-3183

Symes, K. (2017). Orange Is the New Black: the popularization of lesbian sexuality and heterosexual modes of viewing. Feminist Media Studies, 17(1), 29-41. https://doi.org/10.1080/14680777.2017.1261836

Tosenberger, C. (2008a). Homosexuality at the online Hogwarts: Harry Potter Slash Fanfiction. Children's Literature, 36, 185-207. https://doi.org/10.1353/chl.0.0017

Tosenberger, C. (2008b). “Oh my God, the Fanfiction!": Dumbledore's Outing and the Online Harry Potter Fandom. Children's Literature Association Quarterly, 33(2), 200-206. https://doi.org/10.1353/chq.0.0015

Varona Aramburu, D. \& Lara Toledo, P. (2015). "Be ministérico, my friend": diseño de una estrategia transmedia (“Be ministerical, my friend”: design of a transmedia strategy). In C. Cascajosa (Ed.), Dentro de El Ministerio del Tiempo (Inside the Time Ministry) (pp. 203-210). Léeme Libros.

Ventura, R., Guerrero-Pico, M., \& Establés, M.-J. (2019). Ciberactivismo fan lesbiano: acciones de protesta no violenta frente a las representaciones heteronormativas de personajes LGBTQ en televisión (Lesbian fan cyberactivism: nonviolent protest actions against heteronormative representations of LGBTQ characters on television). In A. Farné, \& A. Iranzo-Pastor (Eds.). Comunicación para el cambio social: propuestas para la acción (Communication for social change: proposals for action) (pp. 137-157). Tirant lo Blanch.

Villa-Montoya, M. I., Montoya-Bermúdez, D. \& Escobar, J. (2019). Romance in the femslash. A narrative analysis of fan fiction: Una vez más, una oportunidad más. Anàlisi, 60, 65-79. https://doi.org/10.5565/REV/ANALISI.3192

Waggoner, E. B. (2018). Bury Your Gays and Social Media Fan Response: Television, LGBTQ Representation and Communitarian Ethics. Journal of Homosexuality, 65(13), 1877-1891. https://doi.org/10.1080/00918369.2017.1391015 


\section{SOBRE LOS AUTORES}

MERCEDES HERRERO DE LA FUENTE, Doctora en Ciencias de la Información (UCM, España). Profesora acreditada de la Universidad Nebrija, donde dirigió el Máster en Periodismo Digital y de Datos y el Máster en Periodismo en Televisión. Integra el proyecto I+D+I "Discapacidad y Competencias Digitales en el Sector Audiovisual". Sus líneas de investigación son las nuevas tecnologías y su aplicación en el ámbito audiovisual. Ha sido research fellow en: Cornell University (EE.UU.), Saldford University (Reino Unido), Radboud Univesiteit (Países Bajos) y Karlova Univerzita (República Checa).

iD https://orcid.org/0000-0002-5361-9056

ANTONIO GARCíA DOMínGUEz (ANTO GARZíA), Graduado en Ingeniería Informática (UHU) y Máster en Gestión de Empresas Audiovisuales (EAE Business School). Ha sido Head of Social Media en Atresmedia y actualmente es el responsable de Redes Sociales en la productora audiovisual Fremantle. Sus proyectos destacados son «La casa de papel», «La Voz», «Mask Singer», «Tu cara me suena», «\#Luimelia»y «Got Talent España». Profesor en las universidades Nebrija y Carlos III. Autor de artículos sobre uso y medición de resultados de acciones realizadas en redes sociales.

iD https://orcid.org/0000-0003-2686-0503

MARÍA JOSÉ ESTABLÉS HeRAS, Doctora internacional en Comunicación (Universitat Pompeu Fabra). Profesora permanente en la Universidad Nebrija. Sus áreas de investigación se centran principalmente en las series de televisión y las comunidades de fans. Sus trabajos han sido publicados en distintas revistas indexadas como Profesional de la Información y Learning, Media and Technology. Actualmente, trabaja en los proyectos competitivos de investigación Mediatized EU (Mediatized Discourses on Europeanization and Their Representations in Public Perceptions) y PLATCOM (Plataformas de comunicación, fuerza de trabajo y aprendizaje informal).

D https://orcid.org/0000-0001-9674-3981 